\title{
Application of Wavelet Spectral Decomposition for Geological Interpretation of Seismic Data
}

\author{
Butorin A. V. \\ Expert Department of Geology and Exploration, LLC “Gazpromneft Science \& Technology Centre” emb. rekiMoyki, 75-79, \\ Saint-Petersburg 190000, Russia
}

\begin{abstract}
Modern oil industry is on the way of complication of the geological structure of the deposits. This trend requires specialists to use the latest technologies to analyze available geological and geophysical information. The article describes a new algorithm—continuous wavelet transform in example of synthetic and real data.
\end{abstract}

Key words: Seismic interpretation, spectral decomposition, RGB-blend, Achimov formation.

\section{Introduction}

At present, the development of hydrocarbon deposits is conducted in conjunction with existing seismic data monitoring, which allows predicting geological environment within the target formation. The role of the wave field increases in course of time due to the need to involve in the development more and more complex built deposits with variable non-uniform internal structure, both laterally and vertically. This need is related primarily to proportional decreasing of relatively "simple" in terms of geology, structural deposits, and transition to complex lithological traps. Achimov layer of the Neocomian section in Western Siberia is an example of this type of geological objects in the Russian oil and gas industry, whose accumulation is attached to the bottom of shelf terraces. Deposits in Achimov complex are primarily controlled by local areas of rapid sedimentation-accumulation zones of sedimentary materialsbrought out from shelf areas. Distribution collectors with this type of sedimentation are irregular by area, making its forecast in the interwell space impossible without 3D seismic data [1].

Corresponding author: Butorin A. V., M.S. (geophysics), postgraduate student, research fields: seismic interpretation, spectral analysis.
In addition to the areal irregularity such collectors are distinctive also with relatively low thickness, which imposes restrictions on usage of standard approaches in seismic data analysis. The wave field has certain resolution limit caused by band-limited frequency spectrum of seismic signals. The maximum vertical resolution is accepted to be 1/8-1/4 of the dominant wavelength [2], which is $10-20$ meters in average. Based on these limitations, Achimov collectors are in general objects stipulating the wave field interference, i.e. the imposition of reflections from the top and bottom of the reservoir. Interference nature of the wave field is also a negative factor for seismic data interpretation influencing the amplitude resolution.

A large number of limitations in the interpretation of the wave field lead to increasing of dynamic interpretation methods, which are an attempt to solve the classic problem of geophysics-determining environmental properties from the observed field parameters such as time, amplitude, frequency and phase, as well as their derivatives. Development of algorithms for dynamic interpretation is directly connected to the development of mathematics and software for digital signal analysis.

Spectral representation of the wave field becomes increasingly important in modern seismic exploration. This approach to the dynamic parameters analysis is 
possible due to the development of spectral decomposition technology, i.e. decomposition of seismic data into frequency components [3]. Within this article we will show the possibility to use this technique for predicting reservoir properties both for model and real data sets.

\section{Materials and Methods}

\subsection{Theory of Spectral Decomposition}

Frequency decomposition methods appeared in the end of XX century. One of the first works in seismic interpretation was an article [4], which discussed some features of Fourier window transform application (Gabor transformation) for geological interpretation. Around the same time, the method of CWT (continuous wavelet transform) appeared which later used very widely in many different scientific and practical fields.

Currently, there is insufficient knowledge regarding the processes causing spectral anomalies. Understanding of causes and patterns of observed phenomena within application of the CWT method will give an opportunity to use this algorithm more competently and intelligently for the wave fields' analysis.

The research described in this paper considers frequency-dependent effects observed in the results of CWT for synthetic wave field, calculated on the basis of a given model. Observed regularities in change of spectral parameters make it possible to determine basic conditions and patterns of spectral anomalies, and also to make some assumptionson optimal blending scheme of results visualization for CWT method.

Spectral decomposition method allows us to decompose the original wave field into separate amplitude-frequency components. The described methods are based on the assumption that local studying of the wave field spectrum will provide more information on internal structure of geological objects.

This algorithm was significantly developed since appearance of the wavelet transform in the late XX century, connected with works of Grossman and Morlet. Wavelets are short waves with zero integral value and the location on the independent variable axis (time), able to shift along the axis and scaling. Due to the short duration and different scale of wavelet, decomposition allows us to explore local temporal features of non-stationary processes occurring in time, which is a significant advantage over the Fourier transform, even in its window modification [5].

The presence of time shift allows us to scan studied signal, i.e. explore it on different time levels. This property of the wavelet transform makes it an instrument for studying dynamic processes, i.e. variable over time.

Scalability allows us to change the dominant frequency, i.e. spectral composition of the wavelet. Smaller scale factor values are used to obtain high-frequency wavelets, larger values of the scaling factor on the contrary lead to signal stretching, which corresponds to the low-frequency wavelets.

Large number of wavelets can be used for CWT method, but the most widely used is Ricker signal with good localization in both time and frequency. Its major positive point is the fact that it is similar with the real seismic signals, which is positive for spectral decomposition results of seismic data [6, 7].

\subsection{Visualization of Spectral Decomposition Results}

As a result of CWT spectral decomposition method, the wave field can be decomposed into a series of cubes describing the amplitude of the frequency harmonics given. Further analysis of the spectral decomposition results is to study amplitudes distribution of different harmonics in area.

One of the most common methods of visualizing CWT results is an RGB color blending. Three different frequencies are applied to the input of the algorithm, for example, vertical, horizontal or stratigraphic slices, or cubes of frequency parameters. According to the algorithm each data array is assigned a color code: red, green or blue. The absence of harmonic amplitude 
represented with black color, and its maximum value-the highest saturation. Further, different color channels are combined together so the resulting array has three amplitude values corresponding to color channel. Resulting color of the discrete is determined by three-dimensional color cube, which describes all colors combining red, green, and blue color channels [8].

\subsection{Interpretation of Color Combination Results on the Basis of a Wedged Out Formation Model}

To study interpretation of RGB-mixing results we performed a composition of a wedged out formation synthetic model with variable acoustic impedance. The model was composed with certain parameters as follows: wedge thickness varies in one direction, acoustic impedance varies in perpendicular direction, and thus the impedance of the enclosing formation is constant above and below the wedge. This model is a good approximation of Achimov formation, where sandstone reservoir intrudes into a relatively homogeneous argillic matrix.

Ricker impulse signal with dominant frequency of $30 \mathrm{~Hz}$ was used for three-dimensional wave field synthesis. In this case, the wave field simulation was carried out considering vertical wave propagation neglecting transient processes at the boundaries and formation of harmonic waves. These assumptions allow us to carry out a pure experiment in the "ideal"

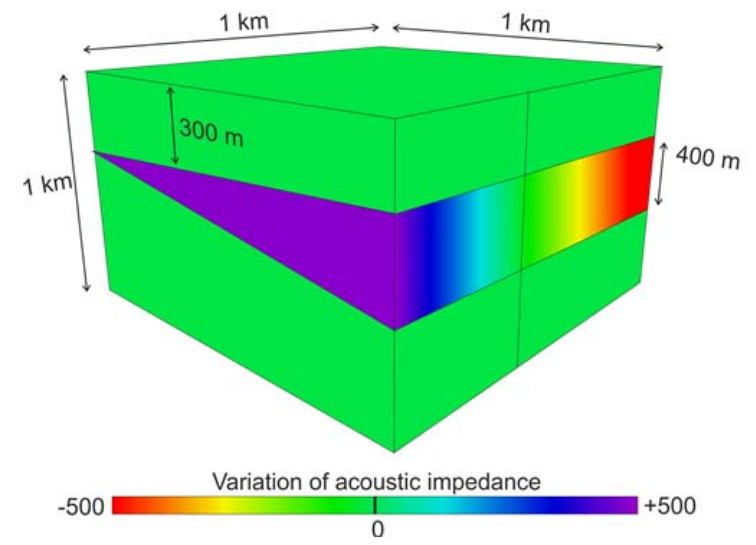

field (Fig. 1).

The resulting synthetic wave field was applied to the input of the CWT algorithm with Ricker signal. The output produced cubes describing frequencies amplitudes within the informative part of the spectrum. For further analysis of the CWT results we studied slices and RGB maps on various slices of the model cube.

\subsection{Studying Influence of Model Interference Effects on Spectral Parameters}

In order to determine characteristics of the resulting spectral anomalies we carried out a detailed analysis of amplitude distribution in frequency cubes. As can be seen from slices in the direction of thinning, maximum anomalies occur at some point prior to the interference of reflections from the top and from the bottom. The brightest anomalies and variations are observed within the interfering layer, weakening to the top and to the bottom (Fig. 2).

In order to determine spectral anomalies patterns we studied amplitude variations of harmonics on slices along the top and in the middle part of the wedge.

First of all we studied amplitude variations of individual harmonics in the middle part of the wedge. For this purpose, the harmonic amplitudes calculated for different acoustic impedance were plotted together on the common graph displaying variation of amplitude versus time thickness of the wedge (Fig. 3).

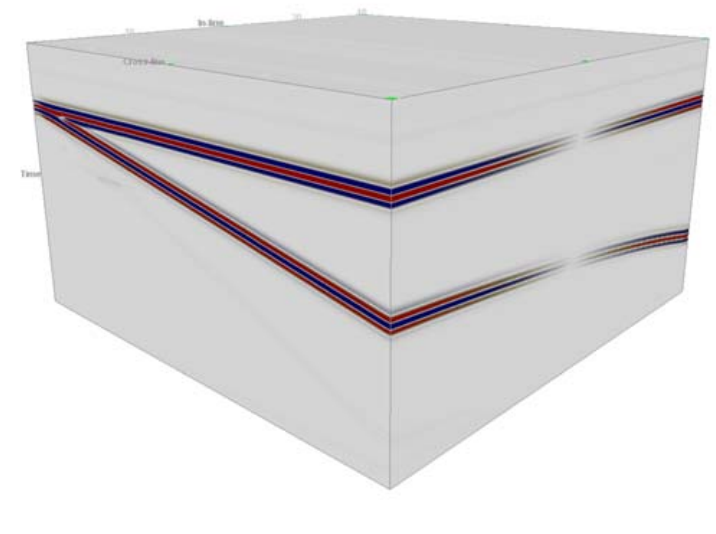

Fig. 1 Model of wedge with variable acoustic impedance (left) and corresponding synthetic wave field (right). 


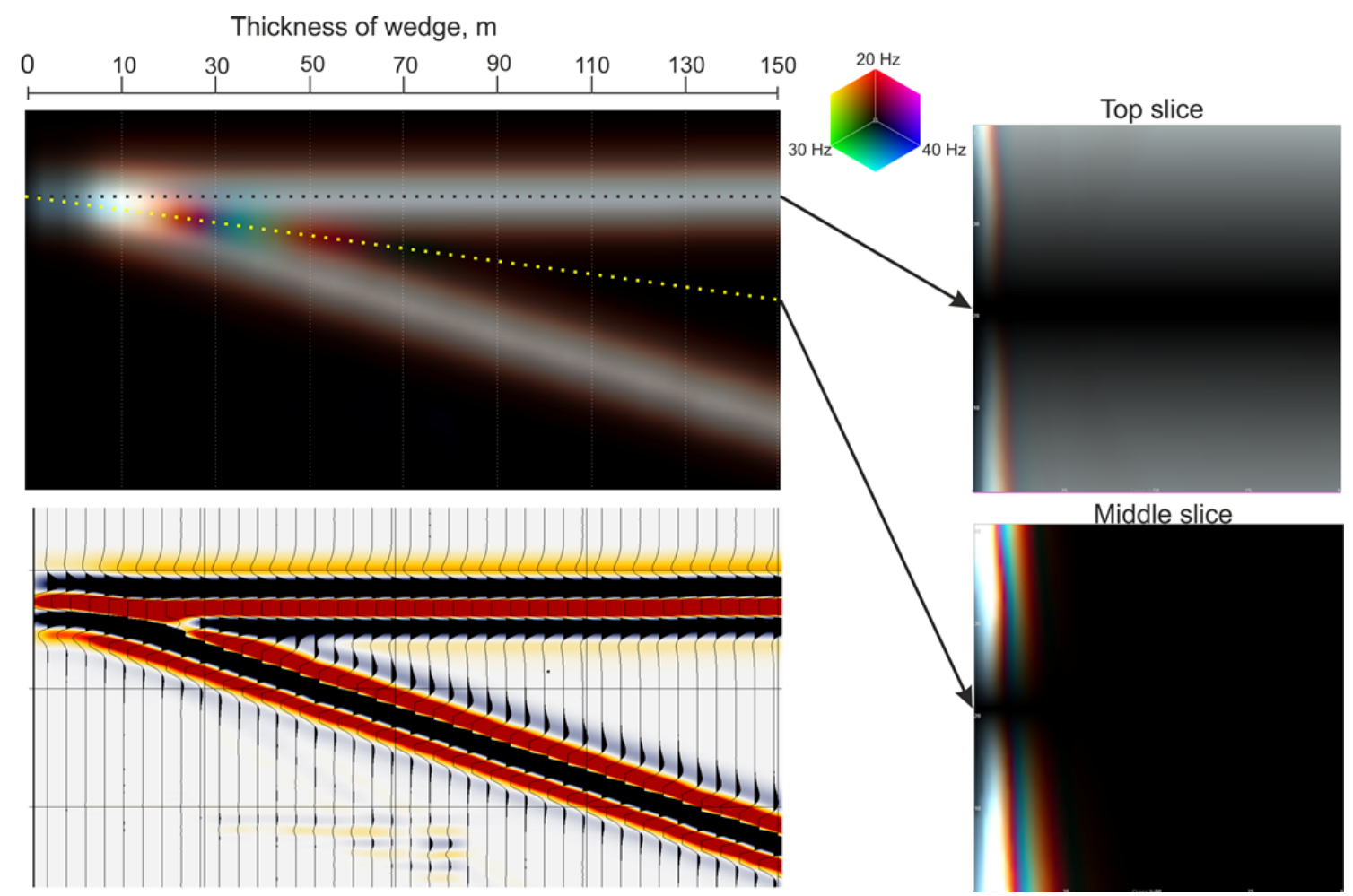

Fig. 2 On the left: RGB section of the wedge and corresponding wave field. On the right: map of spectral blending on the top (top) and in the middle part (bottom) of the wedge.
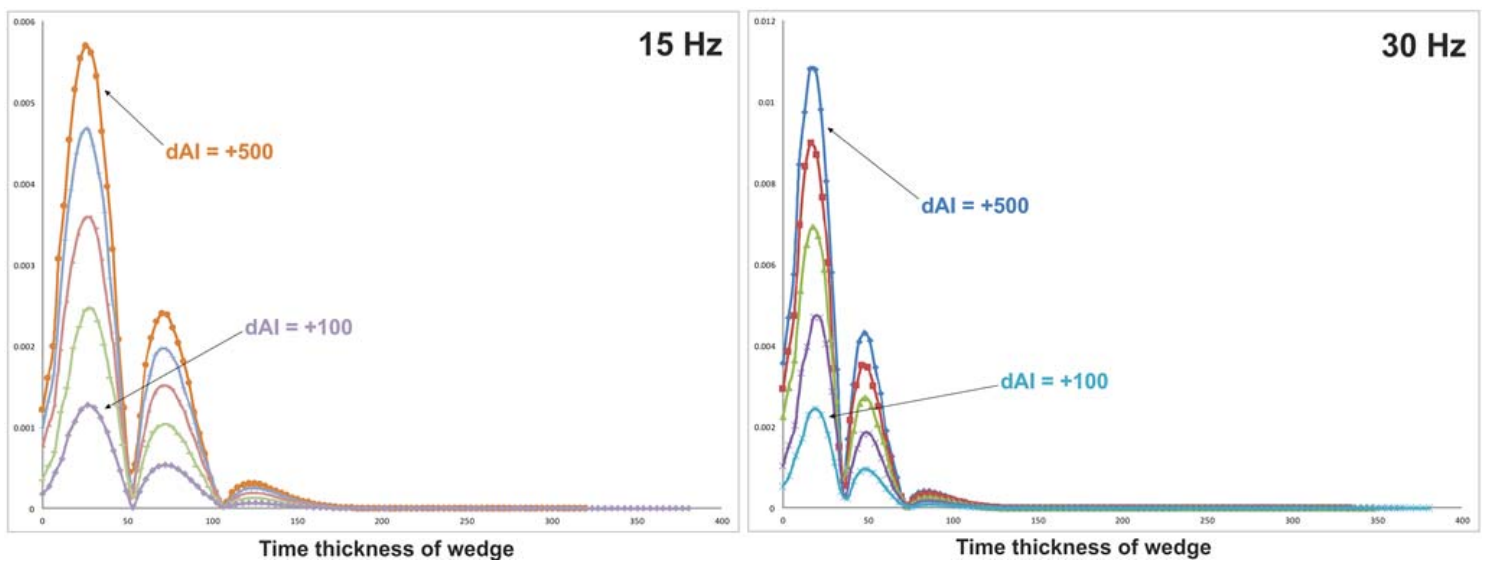

Fig. 3 Harmonic amplitude variation graphs-15 $\mathrm{Hz}$ (on the left) and $30 \mathrm{~Hz}$ (on the right). Color is used to mark curves for different values of relative acoustic impedance inside the wedge (dAI-relative acoustic impedance; $d A I=0$-in clay matrix).

Presence of the interfering layer results in appearance of regular attenuation and amplification of amplitudes of the harmonic waves. Analyzing of the graphs of amplitude characteristics for individual frequencies suggests no effect of acoustic impedance of the interfering layer on variations of amplitudes. The changing of acoustic properties at the boundaries affects only relative amplitude of the spectrum.

Distribution pattern of the anomalies displays its dependence on the frequency of harmonics, so to study this effect we constructed a two-dimensional spectrum, representing the effect of time thickness of the formation on harmonic amplitudes (Fig. 4).

It should be kept in mind that the result of CWT, in contrast to the Fourier transform, tied to time, so two-dimensional spectrum will be different when considering harmonic amplitudes along different slices. Proceeding from this, we considered a two-dimensional 

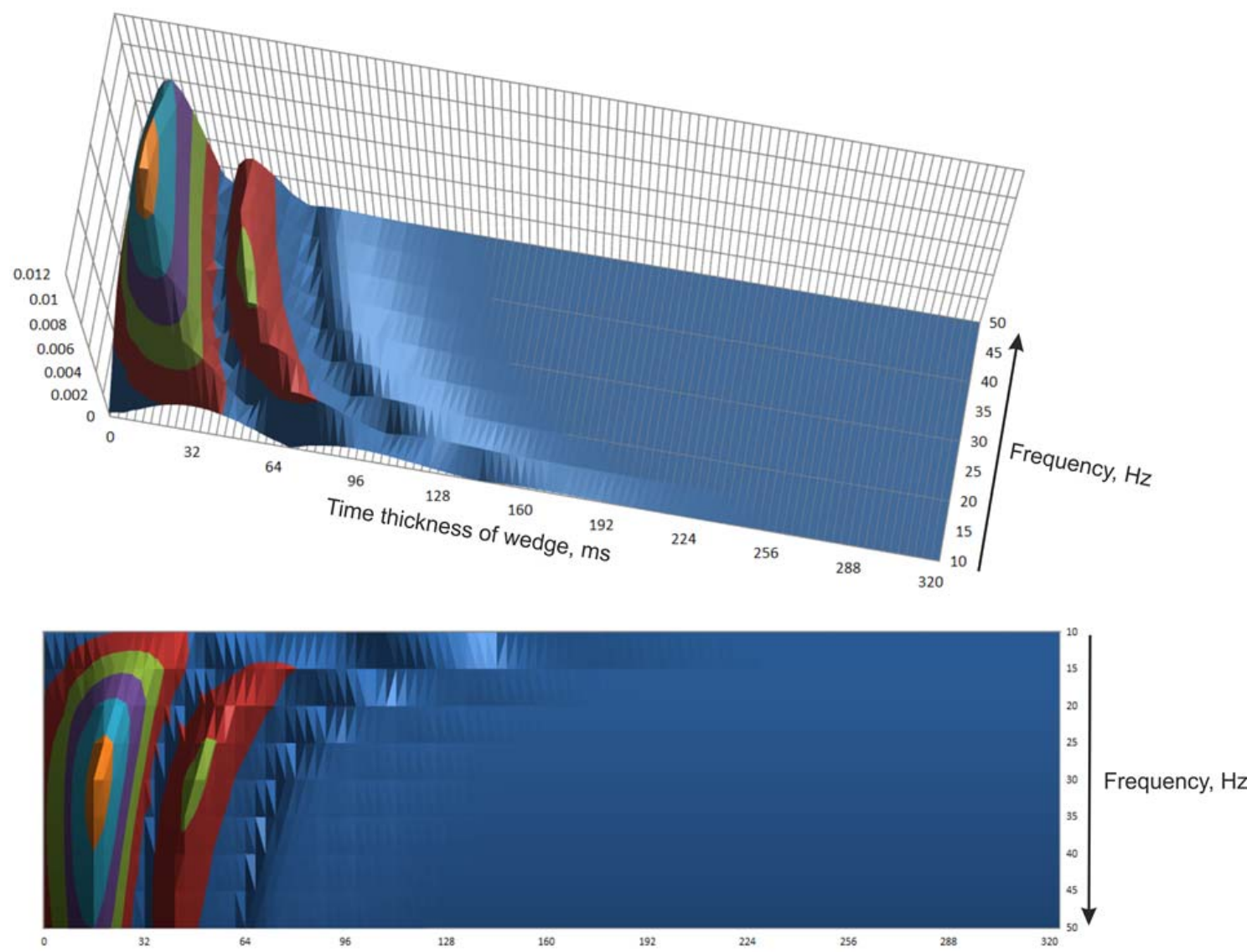

Time thickness of wedge, $\mathrm{ms}$

Fig. 4 Two-dimensional spectra representing amplitude variations of harmonics depending on wedge thickness in the middle part of the wedge.

amplitude spectrum along the reflector top (Fig. 6).

Representation of two-dimensional CWT spectrum is not stationary in reflecting time location of the algorithm;

Using CWT on a spectral slice the origin of amplitude variations is unambiguously fixed. The starting point of spectral anomalies depends on the analyzed frequency, and as a consequence-on the scanning wavelet, as well as on the spatial position of the slice under study. Therefore, occurrence of anomalies in different slices of the cube occurs asynchronously on a temporal thickness. In the middle part of the wedge spectrum anomalies occur at large values of a temporal thickness, compared to the bottom part.

Anomalies of the CWT spectrum have asymmetric amplitudes distribution. The maximum amplitude is observed for the harmonics in the range of the dominant values of the wave field frequency, fading in the lower and higher frequencies.

\subsection{Model Data Application to Determine Optimal Combination of Colors}

Carried out pattern analysis of frequency parameters variations allows to determine the most optimal color scheme for spectral data mixing in the RGB algorithm. For this purpose, based on model data, harmonics amplitude variations were graphed depending on temporal thickness. Analysis of the obtained graphs allows us to make assumptions on the nature of color anomalies of the resulting RGB map. These assumptions have been tested against real data collected on one of the deposits in Western Siberia.

Damping periodicity of harmonic amplitude 
parameters stipulates different results in color mixing. Constructing an RGB map it is necessary to ensure maximum color differentiation of objects, as well as detailing of the resulting map.

Color differentiation is stipulated by combination of extreme amplitude values of the graph. It should be kept in mind that difference in the position of peaks decreases with increasing frequency-difference in the position of anomalies depending on the time thickness of the layer varies sharply at low frequency for close harmonics, difference in the amplitude parameters levels at higher frequency.

Detailing of the resulting map is determined by the maximum frequency of the harmonic wave used for the RGB mixing. Higher frequencies carry more detailed information, whereas lower frequencies are smooth and reflect fewer details. Maximum frequency is restricted by signal/noise ratio which varies depending on the analyzed harmonic wave. Higher frequency is characterized by a predominance of the noise component due to natural damping processes and seismic impulse spectrum, so its use is determined by the quality of the wave field.

Analysis of damping distribution in two-dimensional spectrum leads to the assumption that increasing frequency above $40 \mathrm{~Hz}$ makes no sense, since the behavior of anomalies remains approximately the same.
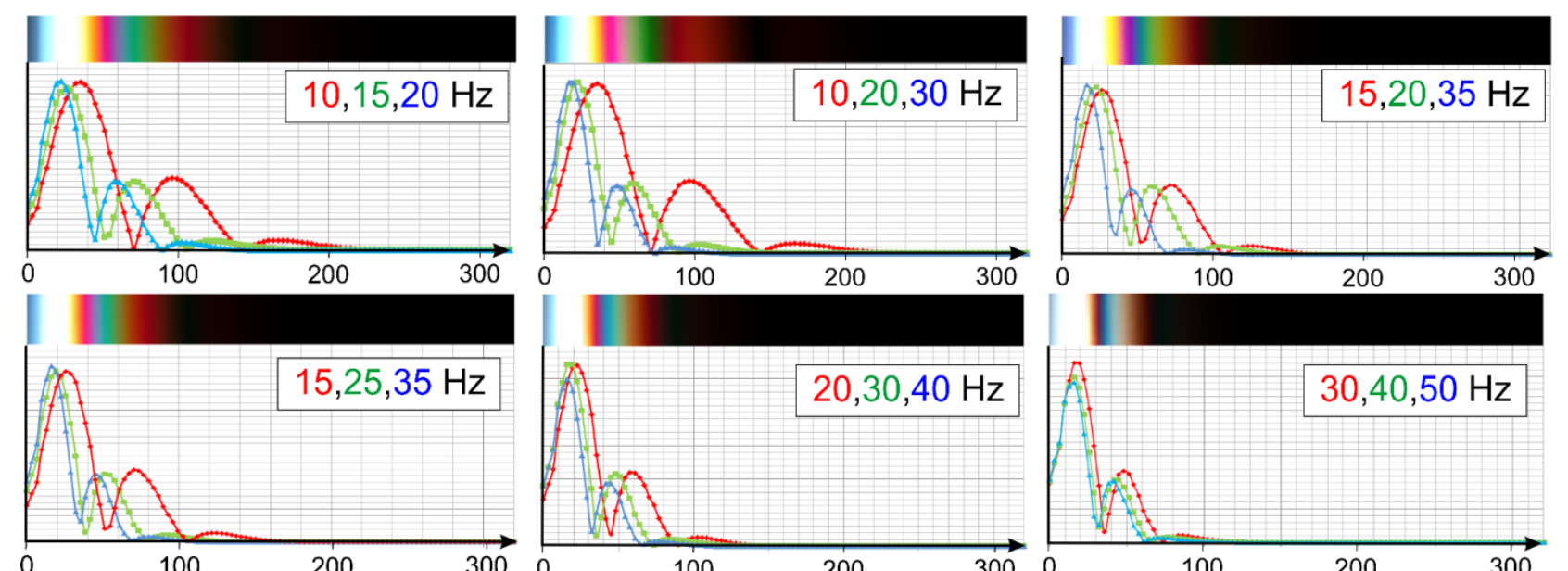

Fig. 5 Color mixing schemes for the RGB algorithm (amplitudes are adjusted to approx. the same level) for different combinations of frequencies (horizontal axis-thickness of wedge, $\mathbf{m}$ ).

The lower frequency during spectral analysis of real seismic data is limited by the boundary of the information availability. In most cases, the lower frequency of the informative part of the spectrum can be accepted at $10 \mathrm{~Hz}$.

Within this study graphs were constructed describing RGB mixing for several frequency combinations: $10-15-20 \mathrm{~Hz}, 10-20-30 \mathrm{~Hz}, 15-20-35 \mathrm{~Hz}$, 15-25-35 Hz, 20-30-40 Hz, 30-40-50 Hz.

Analysis of color mixing of data produced by CWT using different harmonic waves allows us to make several conclusions:

- The use of lower frequency results in a loss of details and predominance of low-frequency background (red areas);

- High frequency components are more informative, but also more affected by noise;

- Due to the wide extrema in the low-frequency part of the spectrum the combination of $10-15-20 \mathrm{~Hz}$ is characterized by low resolution;

- Weak variability of the amplitudes of higher frequency harmonics leads to the fact that their combination in RGB algorithm produces unsatisfactory result—can be seen a considerable reduction of color differentiation;

- The best combinations are $15-25-35 \mathrm{~Hz}$ and 20-30-40 Hz. In these cases, both lower and higher frequencies are combined, all harmonics are in the area

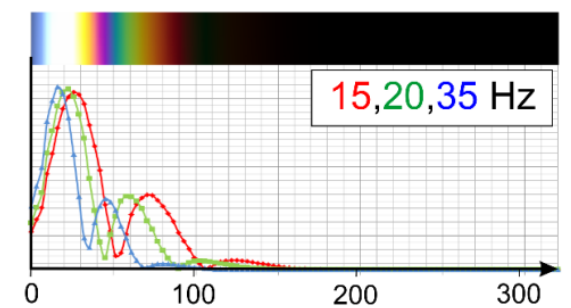




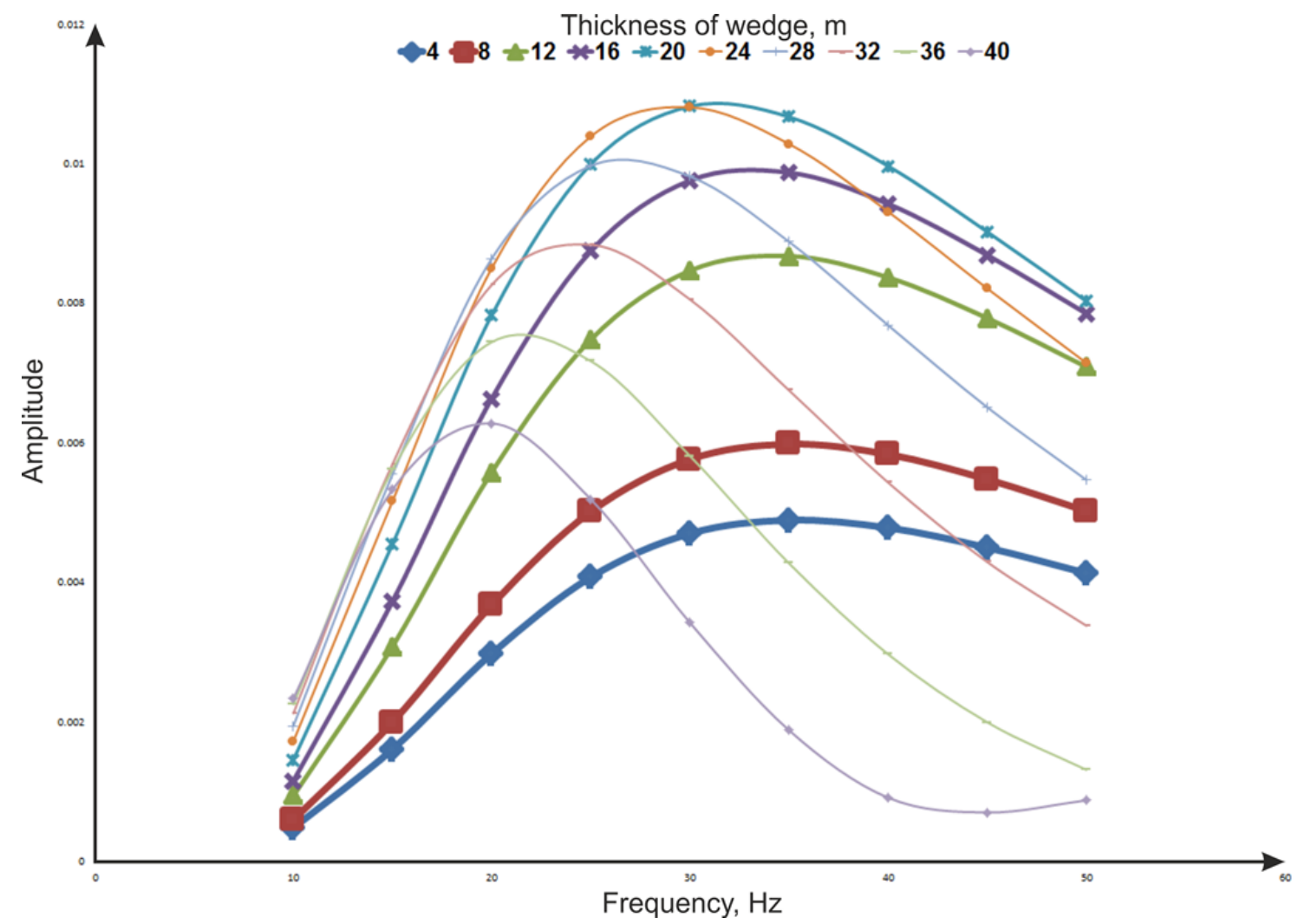

Fig. 6 Graph of harmonics amplitude variations depending on the wedge thickness.

of dominant value, providing a good signal/noise ratio.

\subsection{Estimating the Possibility of Deposit Thickness} Forecast

Having CWT data of the model we are able to analyze the possibility of forecasting thickness of a thin layer according to the spectral parameters of the wave field. Studying the influence of the interfering reservoir thickness was carried out according to the graphs of harmonics amplitude distribution depending on the wedge thickness, and only post interference values were used for the analysis (Fig. 6).

As can be seen from the graphs, the amplitude distribution is subject to certain regularities-the thinner the layer the higher harmonic is dominant. Using this principle allows to estimate theoretically the relative thickness of the formation which caused the appearance of spectral anomalies. Lower thickness of the geological body will be dominant at higher frequency, i.e. RGB-color anomaly will have a blue tint (under normal mixing), relatively thick layers of the interfering body will be characterized by a predominance at lower frequency producing RGB-anomaly with red color.

\subsection{Results of Modeling}

Summarizing the study we can note major results:

- The presence of interfering object in the section leads to a periodic spectral fluctuations;

- Spectral parameters variation depends on position of the slice, along which harmonic waves are studied (top, bottom or middle part of the thin layer);

- The nature of spectral anomalies depends on temporal thickness of the formation and on dominant frequency of the wave field;

- Acoustic impedance of the interfering object does not affect the nature of harmonic amplitude variations;

- Optimal combination of harmonics for the RGB algorithm should include frequencies in the range of dominant values. The most informative combinations of harmonics are up to $40 \mathrm{~Hz}$;

- Color of spectral mixing anomalies may indicate 
thickness distribution within geological bodies-thin areas are characterized by the dominance of higher frequency, relatively thick areas-mainly lower frequency.

\section{Results and Discussion}

\subsection{Practical Application}

Obtained theoretical conclusions on CWT algorithm were extrapolated to studying real formations in Achimov formation attached to the development of submarine fans in one of the deposits in Western Siberia. Application of spectral decomposition allowed increasing informational content of the results of seismic data interpretation. Spectral data allow determining cones geometry, and also the presence of internal channels through which sediment distributed inside the formation. This information is crucial for development planning, as it allows to make an assumption about internal anisotropy of properties within the geologic body.

Fig. 7 displays a comparison of minimal amplitude map to spectral decomposition results in the RGB-mixing visualization. This illustration allows an unambiguous conclusion about the more detailed spectral representation of the wave field. However, it should be noted that the similar anomaly area can be determined on the map of minimal amplitudes as well, but its detailed geological interpretation is not possible.

Application of revealed theoretical regularities in the occurrence of spectral anomalies allows to associate color characteristics of the anomalies distribution with the relative thickness of the fan-higher frequency is predominant in the southern direction, reflecting thinning of the formation.

Studying spectral maps in conjunction with core materials allows on the one hand to confirm correctness of the interpretation made, and on the other with greater confidence to make assumptions about the lithology of undrilled areas.

According to the results of matching of core data with spectral anomalies we can notice high reliability of the RGB map. Well 6 on the spectral map located in the area of development of the supply channel which is unambiguously confirmed by the well section- there is a great conglomerates thickness. Well 5 is located outside of the anomaly area, i.e. attached to relatively deep water facies, this assumption is consistent with core data-mudstones are dominant in the well section. Wells 2 and 1 on spectral maps are located in the main body of the fan, the well slice shows massive sandstones interbedded with conglomerates. Sandstones with massive texture indicate rapid deposition of material, and the presence of conglomerates indicates temporal action of channels. Wells 3 and 4 reveal more distal parts of cones, so the
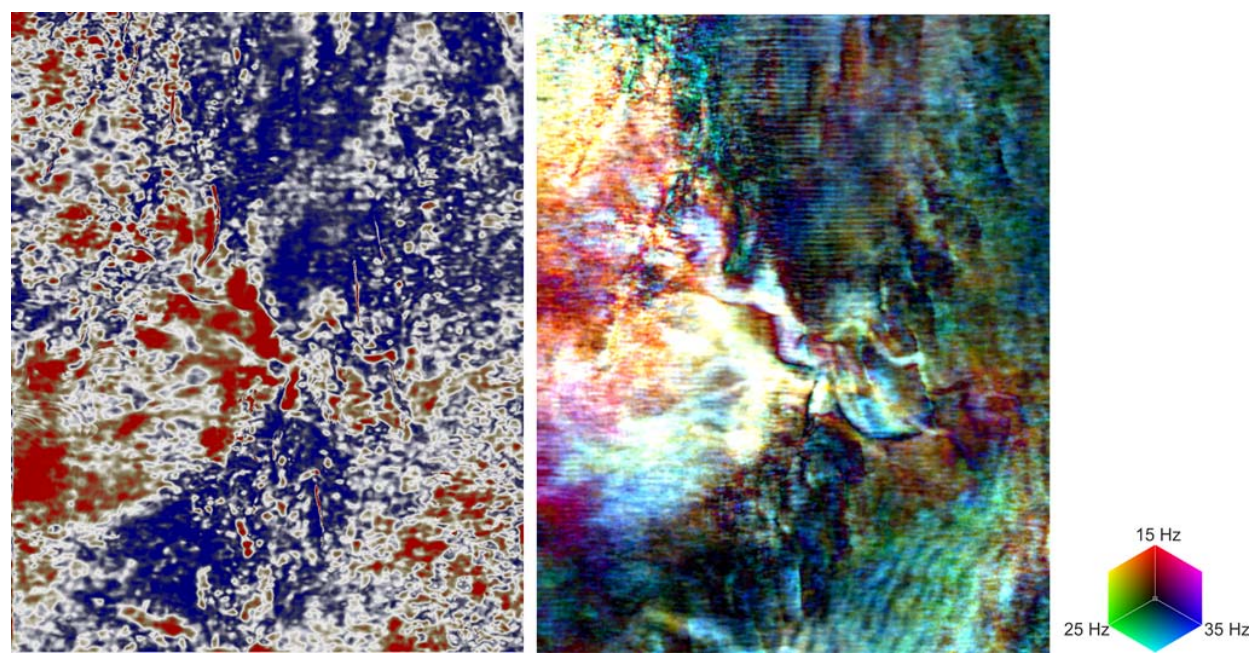

Fig. 7 Comparison of minimal amplitude map (left) and spectral RGB map (right) RGB map analysis in conjunction with core data studying. 


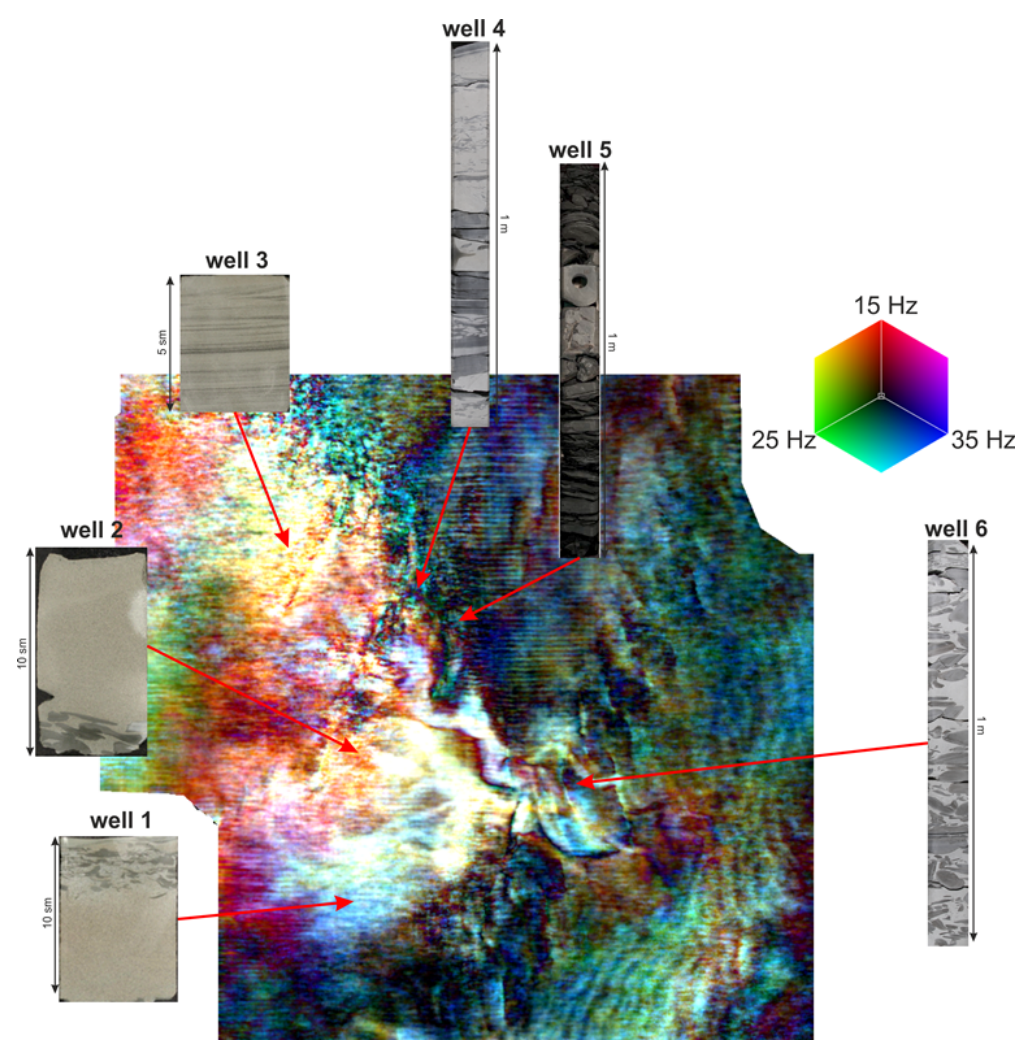

Fig. 8 Matching of spectral map and core materials.

well slice shows thin interbedding of sandstones and mudstones.

Thus, the data obtained through spectral decomposition are fully consistent with the actual core material, which allows to predict facial sedimentation conditions with a high level of confidence.

\subsection{Spatial Assessment of the Fan}

The purpose of the geological modeling is a spatial representation of the geological environment properties (porosity and permeability) for hydrodynamic calculations. In order to obtain correct distribution of the properties in the interwell areas it's necessary to understand geometric parameters of the geological objects. This information may be obtained from seismic data both as two-dimensional (forecast parameters map), and 3D (cubes) representations.

To solve the problem of submarine fan geometrization based on spectral data special objects—geobodies-representing a certain volume of the wave field separated in accordance to a given law were used.

The useful (in terms of the development) volume of seismic data is attached to the spectral anomalies observed on color combination maps. To solve the problem of geometrization of spectral anomalies the RGB-cube is formed, wherein new additional parameter "transparency" is introduced to extract anomalies. Using this parameter we can reduce influence of a certain part of given data.

Setting up transparency parameter is performed on amplitude distribution histograms of corresponding harmonics, separately for each frequency. Since the target is anomaly areas with high amplitude of harmonics, during transparency set up discrete units of lower amplitudes are removed from the analysis.

As a result of application of transparency function to each spectral cube, the resulting RGB-mixing cube is characterized only by abnormal discrete caused by the presence of the interfering body in the section. Synchronous use of three harmonics allows us to study the formation at different scales, thus further evaluate 

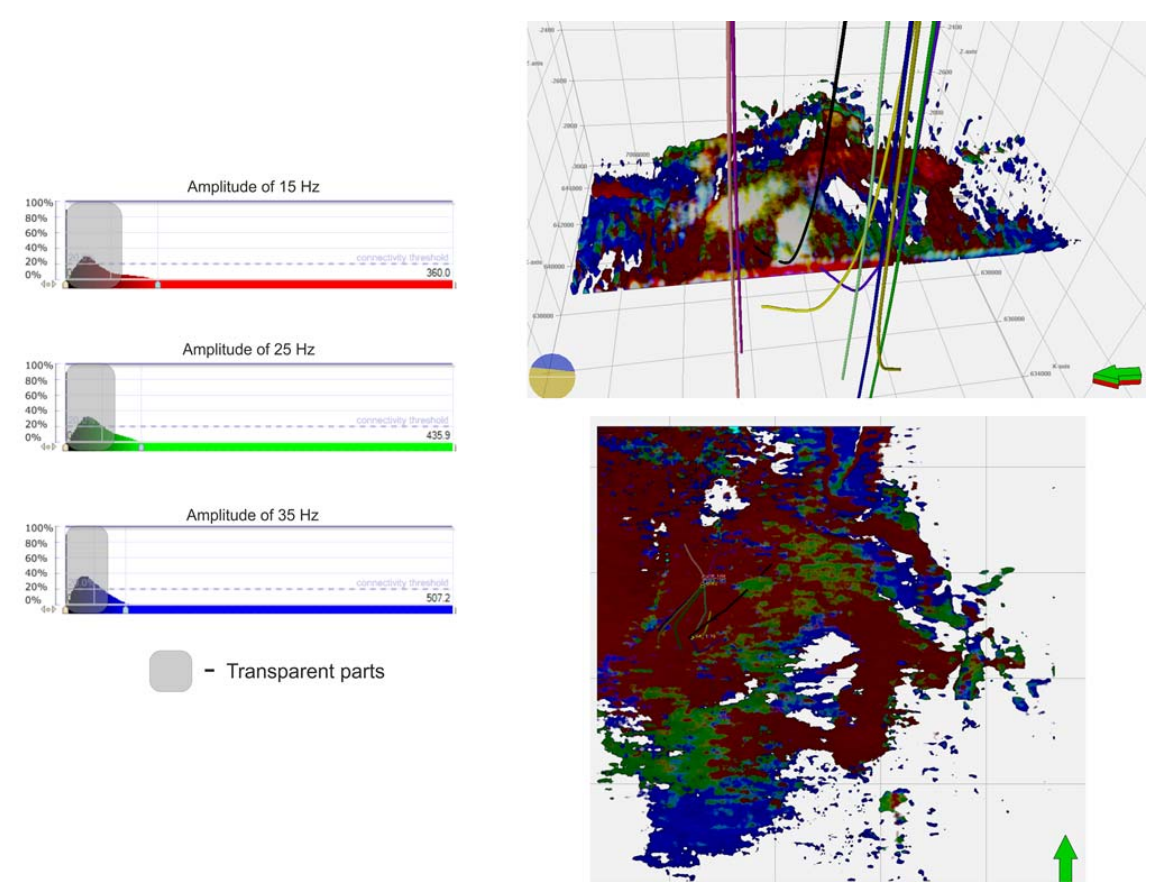

Fig. 9 Algorithm of volume allocation of spectral anomalies. On the left-histogram amplitudes of harmonics with zeroed part, on the right- the result of applying a spatial filter.

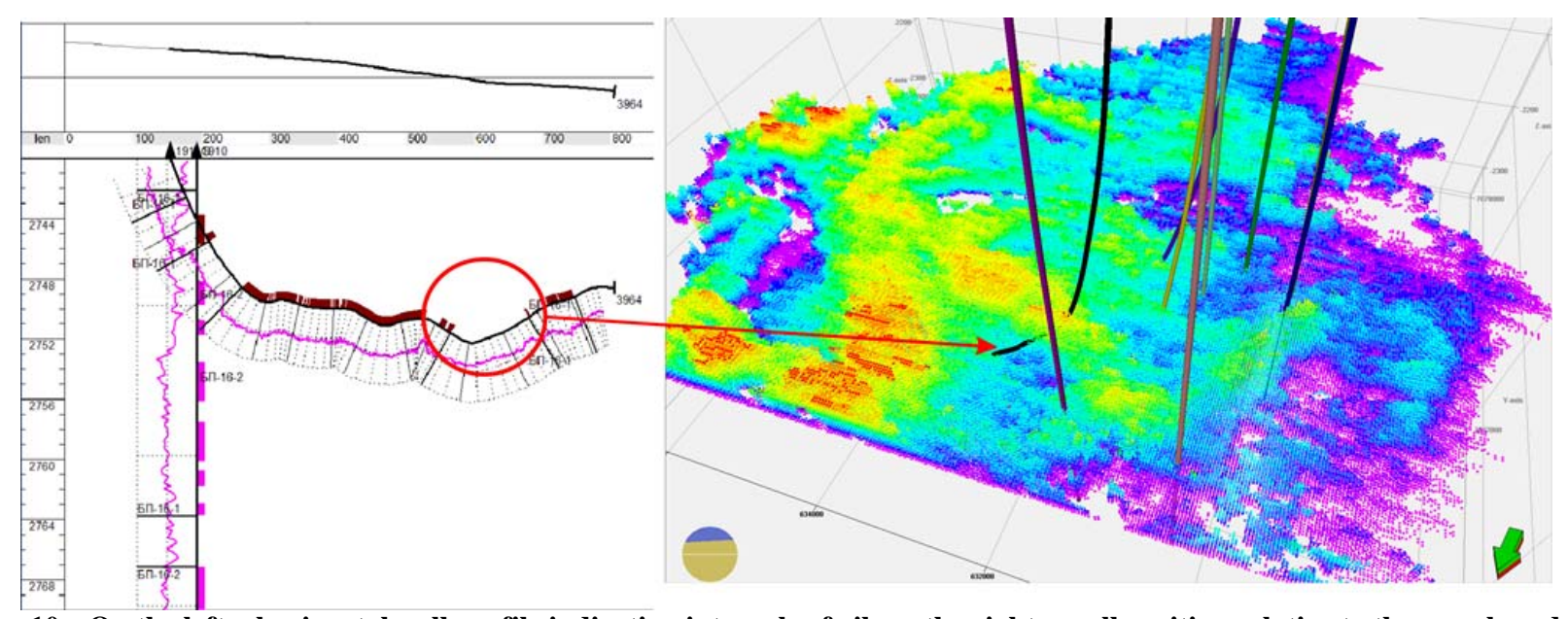

Fig. 10 On the left-horizontal well profile indicating intervals of oil, on the right - well position relative to the cone based on the spectral data.

geometry of the promising object, as can be seen from the figure, the southern distal portion of the fan stands out mainly at higher frequency harmonics (blue).

Discrete anomalies of RGB cube subsequently digitized, i.e. are allocated as spot objects characterized by three spatial coordinates.

This information allows us to construct a more correct geological model, which describes spatial distribution of geological bodies.

Detailing of the obtained spatial representation of the submarine fan allows us to solve the problem of geological modeling, and also to control paths of horizontal wells. Fig. 10 shows the fan system allocated according to the described method with paths of horizontal wells imposed. An extended absence interval of the collector can be observed on some part of the horizontal well. Using obtained estimation of the cone allows to determine that absence interval of the collector corresponds to the outlet of the trunk from productive body. Availability of corresponding 
information prior to drilling will help to avoid such situations in advance and to plan an optimal drilling path.

\section{Conclusion}

This research was carried out to study frequency-dependent effects observed on the wave field in the presence of the interfering body. Basic regularities were considered by the example of a synthetic model of the wedge, which is a good approximation of the collector model in Achimov complex.

Studying of the synthetic model revealed that the nature of spectral anomalies is not connected to petrophysical properties of the formation, but in turn reflects the level of interference on its top and bottom. Constructed two-dimensional spectra of the model allow us to determine a regular variation of harmonics depending on the temporal thickness of the collector, thereby determine the informative part of the spectrum. The behavior of the amplitude parameters of harmonics allows the assumption that the relative thickness of the interfering object-dominant frequency increases with reducing the thickness, which is reflected in the results of the RGB-mixing.

Obtained theoretical conclusions are successfully extrapolated to solve practical problems allocating productive body associated with the development of submarine fans. Detailing of the results allows highly probable reconstructing of the internal structure of geological bodies, which is a determining factor in development drilling.

\section{References}

[1] Butorin, A. V. 2015. "The Internal Structure of Productive Clinoform Horizon by Seismic Data.” Geofizika 1: 10-8.

[2] Widess, M. B. 1973. "How Thin Is a Thin Bed?" Geophysics 38 (6): 1176-80.

[3] Castagna, J. 2006. "Comparison of Spectral Decomposition Methods.” First Break 24: 75-9.

[4] Partyka, G., Gridley, J., and Lopez, J. 1999. "Interpretational Application of Spectral Decomposition in Reservoir Characterization.” The Leading Edge 18 (3): 353-60.

[5] Dauberchies, I. 2001. Ten Lectures on Waveletes. Izhevsk: RHD, 464.

[6] Vityazev, V. V. 2001. Veyvletanalizvremennyhryadov [Wavelet Analysis of Time Series]. Saint-Petersburg: SPbSTU, 58.

[7] Yakovlev, A. N. 2003. Vvedenie v veyvlet-preobrazovanie [Introduction to Wavelet Transform]. Novosibirsk: NSTU, 104.

[8] Henderson. J., Purves. S. J., and Leppard C. 2007. “Automated Delineation of Geological Elements from 3D Seismic Data through Analysis of Multichannel, Volumetric Spectral Decomposition Data.” First Break 25: 87-93. 